\title{
CAPÍTULO 26: DESENVOLVIMENTO DE UMA FERRAMENTA PARA A PREDIÇÃO DA PRESSÃO DE VAPOR DE ESPÉCIES QUÍMICAS UTILIZADAS NA INDÚSTRIA DE ALIMENTOS
}

\section{CAPÍTULO 26: DESARROLLO DE UNA HERRAMIENTA PARA LA PREDICCIÓN DE LA PRESIÓN DE VAPOR DE ESPECIES QUÍMICAS UTILIZADAS EN LA INDUSTRIA ALIMENTARIA}

\section{CHAPTER 26: DEVELOPMENT OF A TOOL FOR THE PREDICTION OF STEAM PRESSURE OF CHEMICAL SPECIES USED IN THE FOOD INDUSTRY}

\author{
Elyson José Neves dos Santos ${ }^{1}$; João Pedro Ferreira ${ }^{2}$; Thayná Habeck Lucio Silva ${ }^{3}$ Caio Veloso Sátiro ${ }^{4}$; Thibério
} Pinho Costa Souza ${ }^{5}$

\section{RESUMO}

DOI: https://doi.org/10.31692/978-65-88970-17-1.385-402

As equações de estado desempenham um papel importante na engenharia, principalmente na química e de alimentos e assumiram um papel crescente no estudo do equilíbrio de fases de fluidos e misturas de fluidos. Originalmente, as equações de estado eram usadas principalmente para componentes puros. Quando aplicado pela primeira vez a misturas, eles foram usados apenas para misturas de compostos apolares e compostos ligeiramente polares. Os problemas industriais envolvem, na sua grande maioria, o contato entre duas ou mais fases em equilíbrio. Consequentemente, o desenvolvimento de tais processos, exige modelos termodinâmicos confiáveis e precisos para representar o equilíbrio entre as fases. Objetivo deste trabalho foi utilizar o EXCEL para implementar um programa para calcular a pressão de vapor de substâncias utilizadas na indústria de alimentos, a partir de uma equação de estado (EdE), utilizando o conceito de isofugacidade no equilíbrio de fases. Foi selecionada a EdE de PengRobinson por ser bem recomendada por autores da área e posteriormente foi comparada com a equação de Antoine para validação dos resultados obtidos da pressão de vapor, do ácido benzoico, etanol e ácido acético. Com o estudo pode-se destacar o uso do Excel para desenvolver um programa como uma alternativa rápida e eficaz para a resolução de equações cúbicas e eficiente para o cálculo da pressão de vapor de espécies químicas, pois calcula e modelar o comportamento de espécies químicas com boa precisão.

Palavras-Chave: Equações de Estado, Peng-Robinson, Excel.

\begin{abstract}
RESUMEN
Las ecuaciones de estado juegan un papel importante en la ingeniería, particularmente en química y alimentos, y han adquirido un papel cada vez más importante en el estudio del equilibrio de fase fluida y mezclas de fluidos. Originalmente, las ecuaciones de estado se usaban principalmente para componentes puros. Cuando se aplicaron por primera vez a mezclas, se usaron solo para mezclas de compuestos no polares y ligeramente polares. La mayoría de los problemas industriales involucran el contacto entre dos o más fases en equilibrio. En consecuencia, el desarrollo de tales procesos requiere modelos termodinámicos confiables y precisos para representar el equilibrio entre las fases. El objetivo de este trabajo fue utilizar EXCEL para implementar un programa de cálculo de la presión de vapor de sustancias utilizadas en la industria alimentaria, basado en una ecuación de estado (EdE), utilizando el concepto de isofuga en el equilibrio de fases. Se seleccionó el EE de Peng-Robinson porque está bien recomendado por los autores en el campo y posteriormente se comparó con la ecuación de Antoine para

\footnotetext{
${ }^{1}$ Engenheiro de Alimentos, Universidade Federal Rural de Pernambuco - UFRPE, elyson_neves@ hotmail.com

2 Mestre em Engenharia de Alimentos, Universidade Federal de Santa Catarina - UFSC, joaoferreira@alimentos.eng.br

3 Mestra em Engenharia de Alimentos, Universidade Federal de Santa Catarina - UFSC, thaynahabeck@gmail.com

${ }^{4}$ Doutor, Universidade Federal Rural de Pernambuco - UFRPE, caiosatiro@ yahoo.com.br

${ }^{5}$ Doutor, Universidade Federal Rural de Pernambuco - UFRPE, thiberio_souza@ hotmail.com
} 
validar los resultados obtenidos a partir de la presión de vapor, ácido benzoico, etanol y ácido acético. Con el estudio, se puede destacar el uso de Excel para desarrollar un programa como alternativa rápida y efectiva para la resolución de ecuaciones cúbicas y eficiente para el cálculo de la presión de vapor de especies químicas, ya que calcula y modela el comportamiento de especies químicas. con buena precisión..

Palabras Clave: Debe contener un mínimo de 3 y un máximo de 5 palabras clave, separadas por comas. Estas son palabras características del tema que sirven para indexar el artículo.

\section{ABSTRACT}

State equations play an important role in chemical and food engineering and a growing role in the balance of fluid phases and fluid mixtures. Originally, the state equations had underlying pure components. Because they were first mixed together, they were only used for mixtures of compounds and polar glassware. Industrial problems most often involve contact between the two phases or more in equilibrium. Consequently, the process of such processes is ideal for thermodynamic models and accurate to represent the balance between phases. The objective of this work was to implement a program to calculate the water vapor pressure in an electric power machine, using state-of-the-art technology. Peng-Robinson's EoS was selected because it was welcomed by area authors and compared with Antoine's equation to obtain optimum results of vapor pressure, benzoic acid, ethanol and acetic acid. With the study one can distinguish the use of Excel to develop a program as a quick and effective alternative to a resolution of cubic equations and efficient for calculation of vapor pressure, good accuracy.

Keywords: Equation of Station, Peng-Robinson, Excel.

\section{INTRODUÇÃO}

As equações de estado desempenham um papel importante na engenharia química e de alimentos e assumiram um papel crescente no estudo do equilíbrio de fases de fluidos e misturas de fluidos. Originalmente, as equações de estado eram usadas principalmente para componentes puros. Quando aplicado pela primeira vez a misturas, eles foram usados apenas para misturas de compostos apolares, (SOAVE, 1972; PENG e ROBINSON, 1976) e compostos ligeiramente polares (HURON et al., 1978; ASSELINEAU et al., 1978; GRABOSKI e DAUBERT, 1978). Posteriormente, as equações de estado se desenvolveram rapidamente para o cálculo do equilíbrio de fases em misturas polares e não-polares. Há muitas vantagens em usar equações de estado para cálculos de equilíbrio de fase. Equações de estado podem ser usadas tipicamente em amplas faixas de temperatura e pressão, e elas podem ser aplicadas a misturas de diversos componentes, variando de gases leves a líquidos pesados. Eles podem ser usados para calcular os equilíbrios vapor-líquido, líquido-líquido e equilíbrio de fluido supercrítico sem quaisquer dificuldades conceituais. O cálculo do equilíbrio de fases foi discutido extensamente por outros autores (SADUS, 1994; SANDLER, 1994; ECONOMOU e DONOHUE, 1996; DIEFENBACHER e TÜRK, 2001; HRNČIČ, 2018).

A equação de estado de van der Waals foi a primeira equação a prever a coexistência líquido-vapor. Mais tarde, a equação de estado de Redlich-Kwong (REDLICH e KWONG, 1949) melhorou a precisão da equação de van der Waals introduzindo a dependência da 


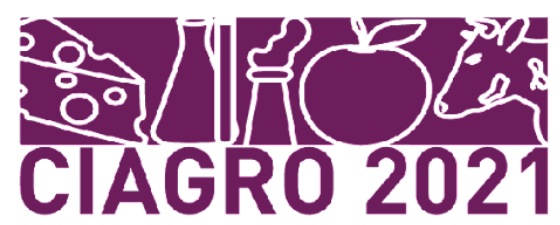

temperatura para o termo atrativo. Soave (1972) e Peng e Robinson (1976) propuseram modificações adicionais para prever com mais precisão as relações de pressão de vapor, densidade líquida e equilíbrio. Carnahan e Starling (1969) e Boublik (1981) modificaram o termo repulsivo da equação de estado de van der Waals para obter expressões precisas para a repulsão do corpo duro. Outros autores (CHEN e KREGLEWSKI, 1977; CHRISTOFORAKOS e FRANCK, 1986; HEILIG e FRANCK, 1989) modificaram os termos atrativos e repulsivos da equação de estado de van der Waals.

Sendo assim, o principal objetivo deste trabalho é utilizar o EXCEL como ferramenta para encontrar a pressão de vapor de substâncias utilizadas na indústria de alimentos, a partir de uma equação de estado (EdE), utilizando o conceito de isofugacidade no equilíbrio de fases.

\section{REFERENCIAL TEÓRICO}

\section{Equações de estado (EdE)}

Em situações da engenharia química ou de alimentos, muitas vezes é o valor de uma propriedade é necessário, mas não existem valores experimentais para essas condições exatas (composição, pressão, temperatura). Quando essas situações surgem, torna-se crucial ter uma maneira adequada de estimar ou prever esses valores. Existem basicamente três maneiras de proceder: base teórica, empírica ou semi-empírica. As equações de estado desempenham um papel importante na engenharia e assumiram um papel crescente no estudo do equilíbrio de fases de fluidos e misturas de fluidos. Originalmente, as equações de estado eram usadas principalmente para componentes puros. Quando aplicado pela primeira vez a misturas, eles foram usados apenas para misturas de compostos apolares, (SOAVE, 1972; PENG e ROBINSON, 1976) e compostos ligeiramente polares (HURON et al., 1978; ASSELINEAU et al., 1978; GRABOSKI e DAUBERT, 1978). Posteriormente, as equações de estado se desenvolveram rapidamente para o cálculo do equilíbrio de fases em misturas polares e nãopolares. Há muitas vantagens em usar equações de estado para cálculos de equilíbrio de fase. Equações de estado podem ser usadas tipicamente em amplas faixas de temperatura e pressão, e elas podem ser aplicadas a misturas de diversos componentes, variando de gases leves a líquidos pesados. Eles podem ser usados para calcular os equilíbrios vapor-líquido, líquidolíquido e equilíbrio de fluido supercrítico sem quaisquer dificuldades conceituais. (SMITH, VAN NESS E ABBOTT, 2007; ATKINS, 2008). O cálculo do equilíbrio de fases foi discutido extensamente em outros lugares (SADUS, 1994; SANDLER, 1994; ECONOMOU e DONOHUE, 1996; DIEFENBACHER e TÜRK, 2001; HRNČIČ, 2018). Quando aplicada a 


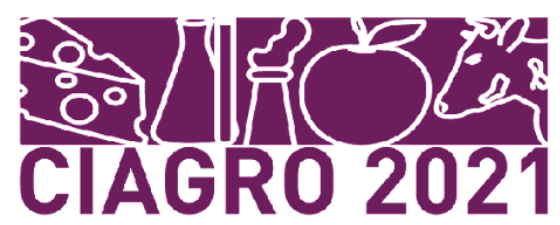

gases reais, a equação do gás ideal pode apresentar grandes desvios. Por essa razão, é prática comum introduzir dados experimentais para melhorar a precisão dos resultados previstos. As equações resultantes são relações empíricas e semi-empíricas. Uma melhoria da relação de gás ideal é a Equação de Estado de van der Waals. Quando assume-se as seguintes ideias:

- O volume total ocupado pelo gás, que coincide com o volume do recipiente para um gás ideal, é reduzido pelo volume ocupado pelas próprias moléculas.

- A pressão exercida pelas moléculas de gás na parede do recipiente é reduzida por causa da atração entre as moléculas devido à sua massa, expressa pelo parâmetro de energia, e aumenta com o quadrado da densidade.

Ao adicionar termos empíricos a equações de estados pode-se refinas os resultados obtidos aproximando-os da realidade. As propriedades volumétricas $(\mathrm{P}, \mathrm{V}, \mathrm{T})$ - são usadas para avaliar as propriedades termodinâmicas e assim calcular as quantidades de calor e trabalho requeridos em processos industriais: Líquidos; Gases e Fluido supercrítico (T > Tc, P > Pc) (SMITH, VAN NESS E ABBOTT, 2007; SANDLER, 1994).

Normalmente representamos as propriedades volumétricas de fluidos puros, graficamente, através de diagramas das Figuras 1 e 2

Figura 1. Diagrama PxT

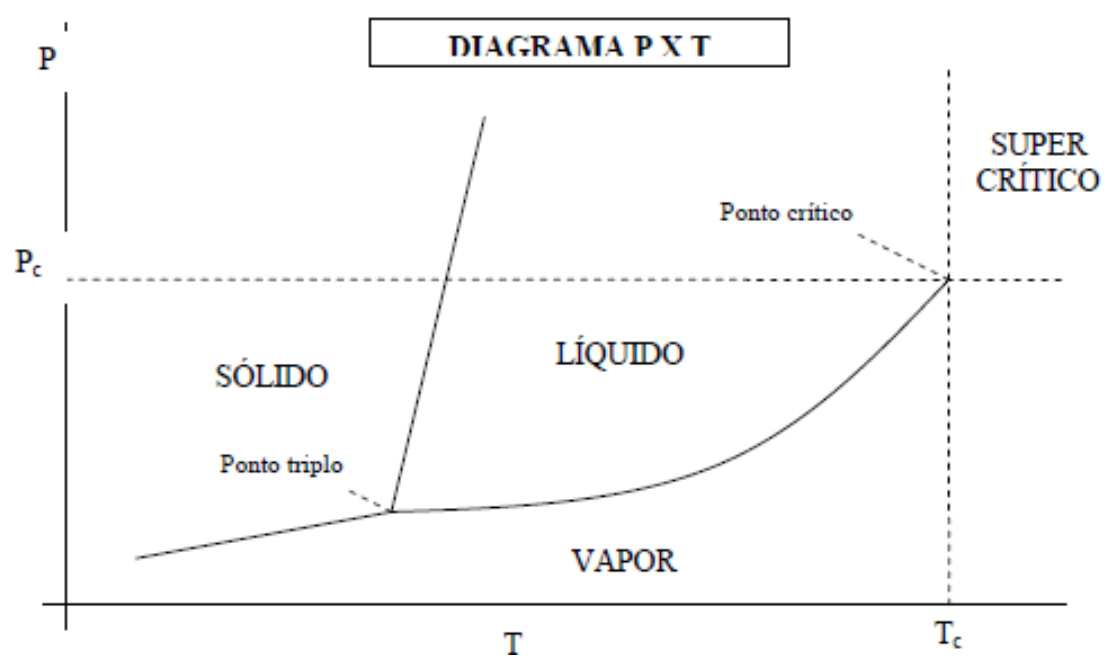

Fonte: Martinez, 2003

No diagrama $\mathrm{P}$ x $\mathrm{T}$ da Figura 1, podemos observar regiões para cada estado físico da substância. As linhas representam as mudanças de estado físico:

- Sublimação (sólido - vapor);

- Fusão (sólido - líquido);

- Vaporização (líquido - vapor).

Também temos o ponto triplo e o ponto crítico, que representam características de cada substância. Podemos usar a regra das fases para mostrar, no diagrama, o número de graus de 
liberdade em cada situação: Quando temos 1 fase, precisamos saber pressão e temperatura para determinar o estado; quando temos mudança de fase, precisamos saber apenas pressão ou temperatura, pois sabemos que o ponto está sobre a linha de mudança de fase; no ponto triplo, não precisamos de nenhuma informação. O ponto já está determinado (SMITH, VAN NESS E ABBOTT, 2007; ATKINS, 2008 e SANDLER, 1994).

Para representar graficamente dados de volume, usamos geralmente o diagrama $\mathrm{P}$ x V (Figura 2):

Figura 2. Diagrama PxV

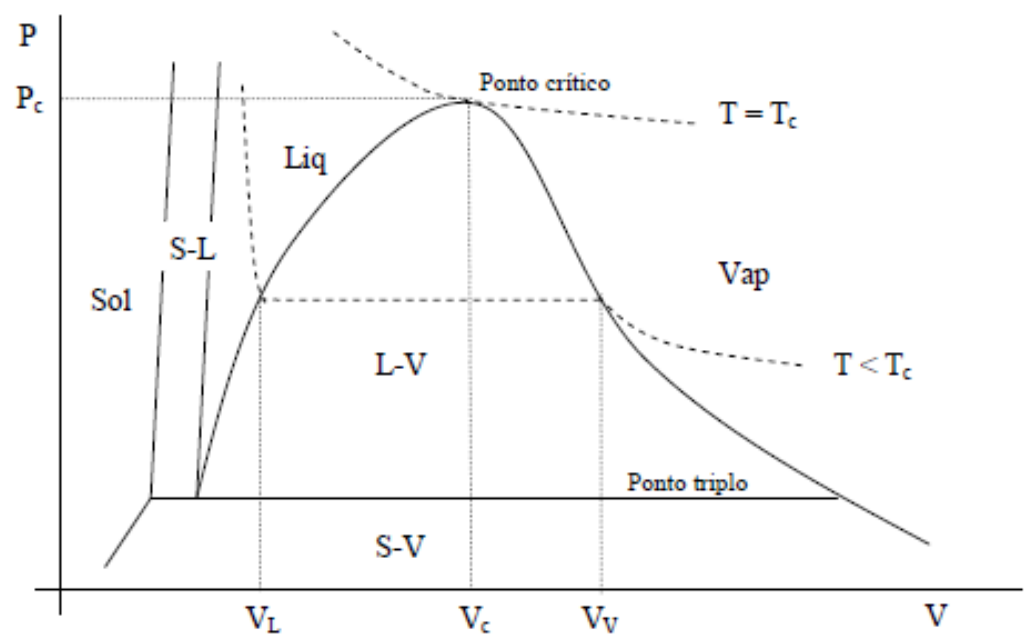

Fonte: Martinez, 2003

Diferentemente do que ocorre no diagrama $\mathrm{P}$ x T, no diagrama $\mathrm{P}$ x V as mudanças de fase são representadas por regiões no diagrama, e não por linhas - pois o volume específico (V) muda durante uma mudança de fase, enquanto a temperatura permanece constante. Da mesma forma, o ponto triplo é representado por uma linha. O volume específico de uma substância no equilíbrio líquido-vapor depende da quantidade relativa de cada fase - é possível determinar o volume específico de cada fase separadamente $\left(V_{L}\right.$ e $V_{V}$ ) (SMITH, VAN NESS E ABBOTT, 2007; SANDLER, 1994).

Da mesma forma, é possível determinar a quantidade relativa de cada fase, em massa, conhecendo o volume específico da mistura líquido-vapor. As curvas tracejadas representam isotermas, onde a temperatura é constante e a pressão varia com o volume. Observamos que na mudança de fase entre líquido e vapor, caso a temperatura esteja abaixo da crítica, tanto pressão como temperatura permanecem constantes. Acima do ponto crítico, onde não há distinção entre líquido e vapor, a pressão sempre diminui à medida que o volume específico aumenta, nas isotermas. No ponto crítico a isoterma $\mathrm{P} x \mathrm{~V}$ apresenta um ponto de inflexão: a derivada segunda de P(V) é zero (ATKINS, 2008; SANDLER, 1994). 


\section{Pressão de vapor}

A pressão de vapor é uma medida da volatilidade de uma substância. Substâncias com alta pressão de vapor são mais voláteis, enquanto que substância com baixa pressão de vapor são substância pouco voláteis. A volatilidade por sua vez depende da natureza química da substância e está intimamente relacionada com as forças que mantêm as moléculas unidas, forças de Van der Waals. Substância que apresentam fortes interações intermoleculares, como ligações de hidrogênio terão pressão de vapor baixa e, portanto, baixa volatilidade. Por outro lado, substâncias que interagem de forma fraca, por forças de dispersão de London, por exemplo, terão pressão de vapor maior e logo serão mais voláteis (MARTINEZ, 2003, ATKINS, 2008).

A pressão de vapor depende fortemente da temperatura do sistema, quanto maior a temperatura maior será a pressão de vapor do composto. Isso é coerente com nossa noção de mudança de fase, uma vez que quanto maior a temperatura, mais as substâncias tendem a fase gasosa. Para diversos propósitos é interessante saber prever a pressão de vapor de substâncias a qualquer temperatura (SMITH, VAN NESS E ABBOTT, 2007; ATKINS, 2008).

Para estimar os valores de pressão de vapor das substâncias duas equações são normalmente utilizadas. A equação de Clapeyron fornece uma conexão vital entre as propriedades de fases a partir de uma relação termodinâmica exata. E a equação de Antoine, que é mais adequada para o uso geral. A equação de Antoine está expressa na Equação 1:

$$
\ln \left(P_{\text {sat }}\right)=A-\frac{B}{T+C}
$$

Equação (1)

A principal vantagem dessa equação é que os valores das constantes A, B e C são facilmente encontrados para um grande número de espécies, cada conjunto de constantes é válido para uma faixa específica de temperaturas, e não deve ser utilizado muito fora dessa faixa. A pressão de vapor de uma substância também pode ser estimada a partir do conceito de isofugacidade entre as fases quando se encontram em equilíbrio. Por sua vez, a fugacidade pode ser encontrada a partir de uma equação de estado (SMITH, VAN NESS E ABBOTT, 2007; SANDLER, 1994).

O conceito de fugacidade(f), que é uma propriedade que pode ser fisicamente medida, e estabeleceu um novo critério de equilíbrio: a igualdade entre as fugacidades, na fase líquida e fase de vapor. A palavra "fugacidade" está baseada em uma raiz latina que significa abandonar, que também é a base para a palavra "fugitivo". Desta forma, fugacidade tem sido interpretada como "tendência para escapar". Quando a tendência para escapar é a mesma nas duas fases, elas estão em equilíbrio. Por exemplo, Para uma espécie pura, fases líquida e vapor coexistindo 
em equilíbrio têm as mesmas T, P e fugacidade (SMITH, VAN NESS E ABBOTT, 2007; SANDLER, 1994).

Origem da fugacidade (f), para uma espécie pura i no estado de gás ideal é:

$$
\mu_{i}=G_{i}^{g i}=\Gamma_{i}(T)+R T \ln P \quad \text { (Gases ideais) } \quad \text { Equação (2) }
$$

Para que a equação acima pudesse ser usada de forma genérica, ou seja, para qualquer gás, Lewis propôs uma nova função, chamada fugacidade (fi), análoga a pressão, dada por:

$$
G_{i}=\Gamma_{i}(T)+R T \ln f_{i} \quad \text { (Gases reais) } \quad \text { Equação (3) }
$$

Essa equação só é válida a T constante. A fugacidade tem a mesma unidade de pressão. Гi $(\mathrm{T})$, a constante de integração a $\mathrm{T}$ constante, é uma função somente de $\mathrm{T}$, que depende da espécie.

As fugacidades de um fluido puro na fase líquida e na fase de vapor são expressas da seguinte maneira:

$$
\begin{array}{lll}
G_{i}^{V}=\Gamma_{i}(T)+R T \ln f_{i}^{V} & \text { (Vapor Saturado) } & \text { Equação (4) } \\
G_{i}^{L}=\Gamma_{i}(T)+R T \ln f_{i}^{L} & \text { (Líquido Saturado) } & \text { Equação (5) }
\end{array}
$$

Por diferença temos:

$$
G_{i}^{V}-G_{i}^{L}=R T \ln \frac{f_{i}^{V}}{f_{i}^{L}}
$$

No equilíbrio:

$$
G_{i}^{V}=G_{i}^{L} \rightarrow G_{i}^{V}-G_{i}^{L}=0
$$

Logo,

$$
R T \ln \frac{f_{i}^{V}}{f_{i}^{L}}=0 \rightarrow f_{i}^{V}=f_{i}^{L}
$$

Esta equação se aplica à mudança de líquido saturado para vapor saturado, ambos na temperatura $\mathrm{T}$ e na pressão de vapor $\mathrm{P}_{\text {sat. }}$ E no equilíbrio quando a fugacidade da fase líquida $\mathrm{e}$ na fase de vapor são iguais. É estabelecida a condição de isofugacidade, critério onde podemos estabelecer que a $\mathrm{P}_{\text {sat }}$ é a pressão de vapor na temperatura especificada (SMITH, VAN NESS E ABBOTT, 2007; SANDLER, 1994).

\section{Aplicações da na indústria de alimentos}

Os problemas industriais envolvem, na sua grande maioria, o contato entre duas ou mais fases em equilíbrio. Consequentemente, o desenvolvimento de tais processos, exige modelos termodinâmicos confiáveis e precisos para representar o equilíbrio entre as fases. Para a modelagem termodinâmica do equilíbrio de fases em sistemas binários ou multicomponentes, através de equações de estado generalizadas, é preciso conhecer as pressões de vapor dos compostos puros que formam a mistura (GEANKOPLIS, 1993). 
Conhecer parâmetros como a pressão de vapor auxilia o engenheiro proporcionar condições de temperatura e pressão global do processo baseados na operação que se deseja realizar como, por exemplo, em processos como destilação, extração de compostos, métodos de separação, além de estimar a melhor condição de funcionamento de bombas para evitar cavitação das mesmas, devido à pressão de vapor do fluido escoado (SMITH, VAN NESS E ABBOTT, 2007; GEANKOPLIS, 1993).

\section{METODOLOGIA}

\section{Seleção da equação de estado}

Inicialmente foram estudadas as equações de estado que são frequentemente citadas na literatura. As equações demonstradas na Tabela 1, são as principais EdE encontradas universalmente nos livros de termodinâmica e em artigos da área.

Tabela 1- Equações de Estado

\begin{tabular}{cc}
\hline Equação & Referência \\
\hline$P=\frac{R T}{V-b}-\frac{a}{V^{2}}$ & Van der Waals (1873) \\
\hline$P=\frac{R T}{V-b}-\frac{a}{T^{1 / 2} V(V+b)}$ & Redlich-Kwong (RK) (1949) \\
\hline$P=\frac{R T}{V-b}-\frac{a(T)}{V(V+b)+b(V-b)}$ & Peng-Robinson (PR) (1976)
\end{tabular}

A partir dessas equações foi selecionada a EdE de Peng-Robinson, por ser mais recomendada por autores da área, a partir da seleção a EdE foi comparada com a equação de Antoine. A Equação de Peng e Robinson (1976), pois suas modificações adicionais preveem com mais precisão as relações de pressão de vapor, densidade líquida e equilíbrio.

\section{Escolha do método de resolução da equação cúbica}

Com a escolha da Equação de Peng e Robinson (1976), estimou-se a pressão de vapor em diferentes temperaturas. Expressar a equação de estado cúbica através do coeficiente de compressibilidade auxilia a construção do programa, uma vez que a equação se torna mais simples de ser manipulada e ser calculada iterativamente. Logo, a partir de um screening feito na literatura, o método de determinação das raízes de uma equação cúbica selecionado foi aquele que apresentou a discretização mais compatível com o problema abordado. 
E para isso escolheu-se o método de Cardano e Tartaglia para encontrar as raízes da equação cúbica, quando a mesma for expressa através do fator de compressibilidade (Z), como demonstrado em Smith, Van Ness e Abbott (2007).

\section{Construção do programa}

Ao escolher a EdE e o método de determinação das raízes, foi construído um programa no Excel, para determinar a pressão de vapor de diferentes espécies químicas de forma iterativa. O programa realiza os cálculos a partir da inserção de parâmetros termodinâmicos fornecidos pelos usuários, respeitando o conceito de isofugacidade, onde no equilíbrio, a fugacidade da fase de vapor e da fase líquida são iguais (SMITH, VAN NESS E ABBOTT, 2007; SANDLER, 1994).

\section{Análise dos resultados e construção dos gráficos}

Após a construção do programa, a validação da sua eficiência foi realizada a partir de uma análise comparativa entre os valores de pressão de vapor obtidos através dos cálculos do programa e os obtidos analiticamente através da equação de Antoine.

Para melhor visualização foram elaborados gráficos comparativos. Nestes, é possível observar a precisão dos cálculos do programa nas faixas de temperaturas aceitáveis para os cálculos de Antoine. Com o auxílio do programa, foram construídos diferentes diagramas PxT para as espécies químicas abordadas.

\section{Escolha das espécies químicas utilizadas na indústria de alimentos}

Os problemas industriais envolvem, na sua grande maioria, o contato entre duas ou mais fases em equilíbrio. Consequentemente, o desenvolvimento de tais processos, exige modelos termodinâmicos confiáveis e precisos para representar o equilíbrio entre as fases. Para a modelagem termodinâmica do equilíbrio de fases em sistemas binários ou multicomponentes, através de equações de estado generalizadas, é preciso conhecer as pressões de vapor dos compostos puros que formam a mistura.

Com isso foram escolhidos os seguintes compostos para estimar suas pressões de vapores em diferentes temperaturas, Tabela 2.

Tabela 2 - Propriedades termodinâmicas do Ácido Benzóico, Etanol e Ácido Acético

\begin{tabular}{cccc}
\hline Substância - Espécie Pura & Tc (K) & Pc(MPa) & Fator Acêntrico $(\omega)$ \\
\hline Ácido Benzoico (C6H5COOH) & 751 & 4,47 & 0,603
\end{tabular}




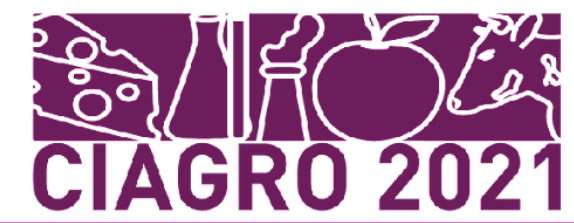

\section{$\begin{array}{llll}\text { Etanol (CH3CH2OH) } & 513,9 & 6,148 & 0,645\end{array}$}

\begin{tabular}{llll}
\hline Ácido acético (CH3COOH) & 592,0 & 5,786 & 0,467
\end{tabular}

Legenda: Tc - Temperatura crítica, Pc - Pressão crítica, Fonte: SMITH, VAN NESS E ABBOTT (2007).

$\mathrm{O}$ ácido benzóico, $\mathrm{C} 6 \mathrm{H} 5 \mathrm{COOH}$, é um composto classificado como ácido carboxílico. E é mais especificamente um ácido fraco e seus sais são usados como conservante de alimentos e naturalmente ocorre em algumas espécies de plantas (FOOD INGREDIENTS BRASIL, 2015). Este ácido atua como germicida na preservação de alimentos, síntese de corantes e também como adjuvante farmacológico. Ele é frequentemente encontrado em bebidas que contém vitamina $\mathrm{C}$, atuando como conservante.

$\mathrm{O}$ etanol (álcool etílico) de formula, $\mathrm{CH}_{3} \mathrm{CH}_{2} \mathrm{OH}$ ou $\mathrm{C}_{2} \mathrm{H}_{6} \mathrm{O}$, é uma substância orgânica obtida da fermentação de açúcares, hidratação do etileno ou redução de acetaldeído. É encontrado em bebidas alcoólicas como cerveja, vinho, aguardente, tequila, Uísque entre outras. O Etanol também é utilizado como combustível de motores de explosão, constituindo assim um mercado em ascensão para um combustível obtido de maneira renovável e o estabelecimento de uma indústria de química de base, sustentada na utilização de biomassa de origem agrícola e renovável (MARTIN et. al, 2013)

Conhecer a pressão de vapor do etanol dá suporte ao processo de destilação, uma vez que, o mosto fermentado possui em sua constituição água e uma mistura de álcoois dos quais o etanol se faz presente e é separado devido o processo de destilação. Logo, uma exigência básica para a separação dos componentes em uma destilação é que a composição do vapor seja diferente do líquido com o qual está em equilíbrio.

O ácido acético é formado pela fermentação através de bactérias acéticas, que ao estarem presentes em cerveja ou vinho ao que ficarem expostas ao ar ambiente, oxidam o etanol presente. $\mathrm{Na}$ indústria de alimentos o próprio vinagre já é um produto bem consumido e dependendo da composição do mosto obtém-se vinagres com propriedades particulares. $\mathrm{O}$ ácido acético é usado amplamente para reduzir o $\mathrm{pH}$, controlar o crescimento microbiano ou como aromatizante. $\mathrm{Na}$ forma pura é pouco usado na indústria de alimentos, porém é largamente empregado na forma de vinagre. (FOOD INGREDIENTS BRASIL, 2015). 


\section{RESULTADOS E DISCUSSÃO}

\section{Interface do Programa}

Na figura 3 é mostrada a interface do programa desenvolvido no Excel. O presente formato foi construído visando uma fácil manipulação pelos usuários, onde os mesmos somente conhecendo as propriedades termodinâmicas da substância que deseja conhecer obtém a pressão de vapor na temperatura. Além da pressão de vapor o programa possibilita o usuário a obter os valores do fator de compressibilidade da fase de vapor de da fase líquida e seus respectivos volumes molares. SANDLER (1994) e DIEFENBACHER (2001), conseguiram implementar uma programação para obter os mesmos dados através do Mathlab®.

Na região em verde o usuário insere as propriedades termodinâmicas da substância estudada, os dados incluem Temperatura crítica (Tc), Pressão Crítica (Pc) e o fator acêntrico. Após inserir as propriedades termodinâmicas o usuário especifica em qual temperatura, área em vermelho, ele quer obter a pressão de vapor da espécie química estudada. E no botão, calcular pressão de vapor, foi vinculado a uma macro que realiza a operação matemática para obedecer ao critério de isofugacidade. 


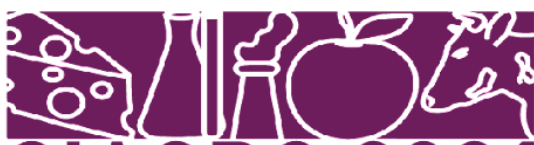 \\ CIAGRO 2021}

Figura 3 - Interface da ferramenta desenvolvida

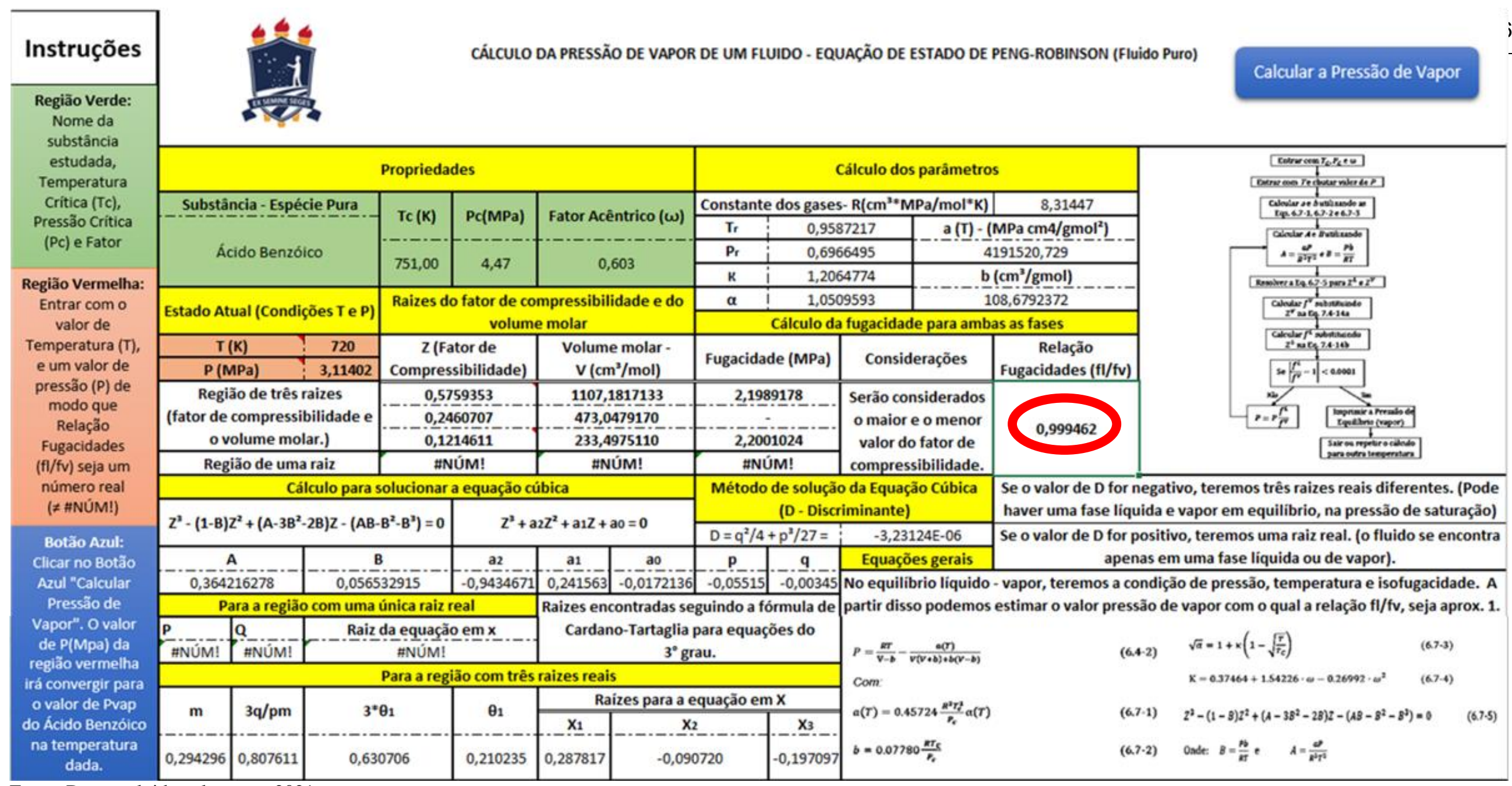

Fonte: Desenvolvida pelo autor, 2021 


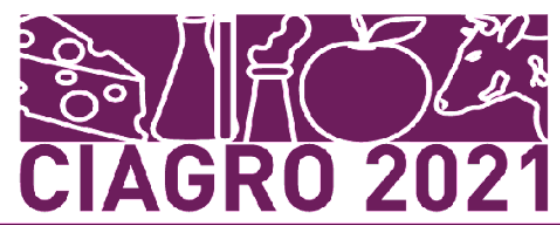

Como foi utilizada a equação de Peng-Robinson (1976), a partir das propriedades inseridas. As equações inseridas nas células foram implementada segundo o método de cardano e Tartaglia para a resolução da equação cúbica expresso no fator de compressibilidade. E logo após a obtenção das raízes o programa utiliza os fatores de compressibilidade da fase de vapor e da fase líquida obtidos, para calcular as respectivas fugacidades. A macro vinculada ao botão, ela segue o procedimento usando-se o recurso do Excel análise de dados na opção teste de hipóteses, na qual é selecionada a ferramenta atingir meta e especifica-se a função objetivo selecionando a célula indicada em vermelho, para calcular iterativamente a pressão de vapor da substância nas condições de entrada, que satisfaça a condição de isofugacidade, em que $\mathrm{fl} / \mathrm{fv} \approx$ 1, ou seja, o programa calcula a pressão de vapor em que a relação das fugacidades sejam o mais próximo possível de 1 .

\section{Validação da predição do diagrama PxT}

Neste tópico serão apresentados os diagramas de Pressão x Temperatura para cada uma das substâncias estudadas, realizando um comparativo entre os valores de Pressão de vapor da substância pura calculado a partir da equação de Antoine (que é o método mais utilizado para estimar este parâmetro) e o programa desenvolvido pelo autor utilizando a equação de PengRobinson (Resolvido a partir do método de Cardano e Tartaglia no software Microsoft Excel).

Os parâmetros da equação de Antoine, assim como as temperaturas mínima e máxima as quais pode-se aplicar a equação para cada substrância, foram obtidos em SMITH, VAN NESS E ABBOTT (2007), e estão apresentadas na Tabela 3.

Tabela 3. Parâmetros da equação de Antoine para as substâncias estudadas e Temperaturas máximas e mínimas

\begin{tabular}{l|cccrr}
\hline \multicolumn{1}{c|}{ Substância } & Ant (A) & Ant (B) & Ant (C) & Tmáx (K) & Tmin (K) \\
\hline Ácido Benzóico & 17,1634 & 4190,70 & $-125,20$ & 560 & 405 \\
Ácido Acético & 16,8080 & 3405,57 & $-56,34$ & 430 & 290 \\
Etanol & 18,9119 & 3803,98 & $-41,68$ & 369 & 270 \\
\hline
\end{tabular}

Equações de estado são usadas frequentemente utilizadas para correlacionar dados e estudar as condições possíveis dos sistemas, em vez de fornecer previsões genuínas. Isto é bem conhecido, pois sabe-se que a equação de van der Waals não pode ser usada para correlacionar com precisão a coexistência vapor-líquido para fluidos. Em contraste, a adição de mais parâmetros ajustáveis e dependência de temperatura para o termo atraente usado nas equações de estado de Redlich-Kwong, Soave-Redlich-Kwong e Peng-Robinson resultam em correlações 
mais precisas da pressão de vapor (ABBOTT, 1979; HAN et al., 1988). E isso é comprovado ao comparar as pressões de vapores das espécies, obtidas via equação de estado de Peng-

Robinson em comparação com a equação empírica de Antoine. Plackov (1995) fala que é possível através dos parâmetros ajustados nas EdE apresentam uma grande melhoria na previsão de pressão de vapor pode ser obtida.

Na figura 4 estão apresentados os diagramas de Pressão de vapor versus a temperatura para o ácido benzoico, Etanol e Ácido Acético. Para a construção do gráfico a faixa de temperatura aceita para o cálculo de Pvap a partir de Antoine (Eq. 1) foi fracionada em 17 temperaturas, as quais foram submetidas ao cálculo de Pvap utilizando-se o programa desenvolvido pelo autor e a equação de Antoine, com os parâmetros apresentados na Tabela 3. 
Figura 4 - Diagrama PxT comparativo para o ácido benzoico, etanol e ácido acético

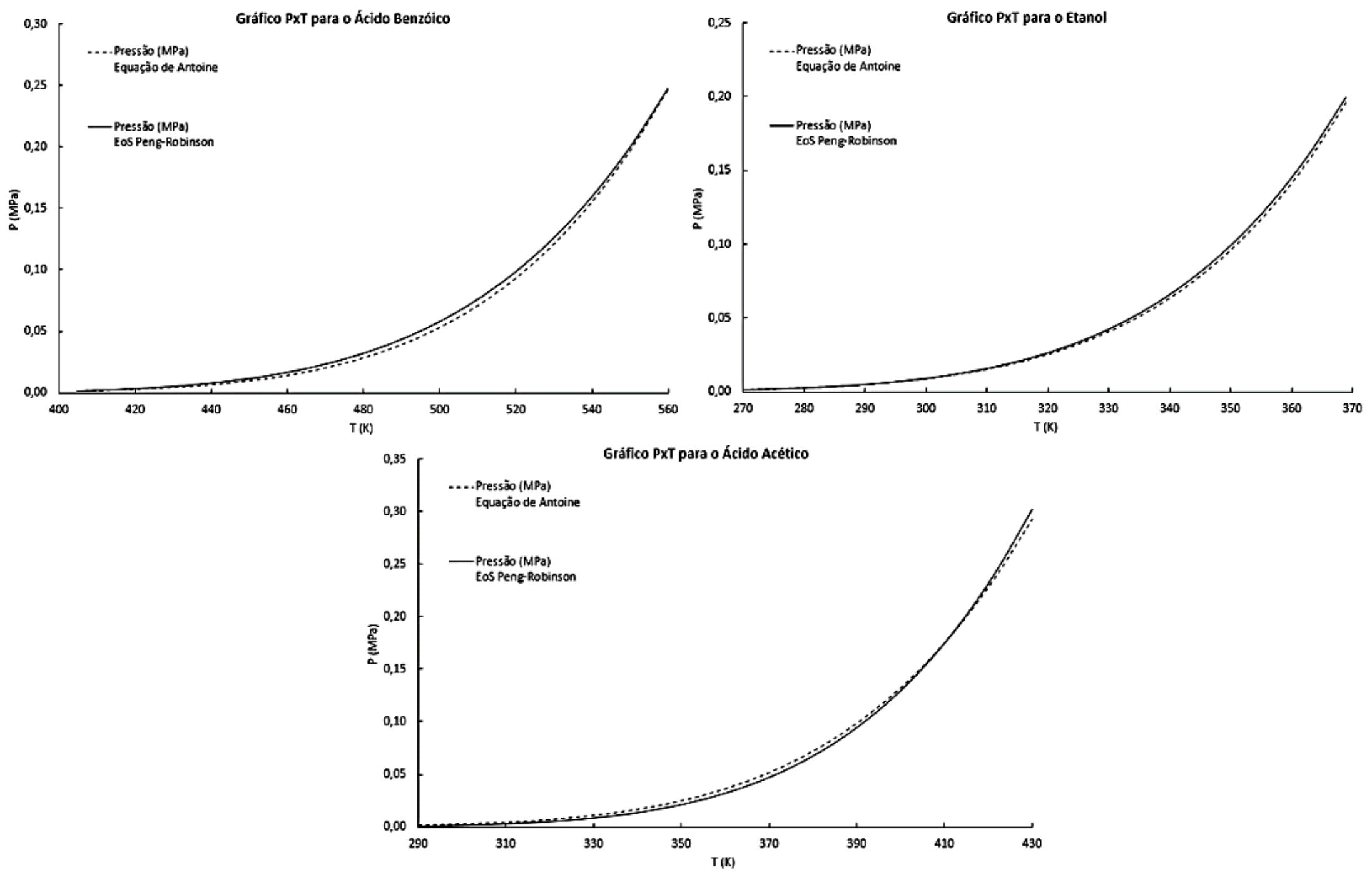


Ao analisar a figura é possível observar que os valores de Pressão de vapor do Ácido Benzóico, etanol e ácido acético obtidos através da simulação no programa desenvolvido no MS Excel apresentam-se muito próximos dos valores calculados a partir da Equação de Antoine, tomada como padrão para a validação do programa.

Para o ácido benzoico a maior diferença entre o programa e o padrão deu-se entre as temperaturas 463,13 e 550,31 K, onde o erro mostrou-se acima de 3 x 10 $0^{-3}$. Mostrando que o programa se mostrou eficaz para o cálculo da pressão de vapor do Ácido Benzóico, com um Somatório dos erros quadrados de 2,6 x 10 $0^{-4}$ o que valida a predição desta propriedade pela ferramenta construída.

Quanto ao etanol a maior diferença entre o programa e o padrão é observada em temperaturas superiores a $350,4 \mathrm{~K}$, onde o erro é superior a $3 \times 10^{-3}$. O Somatório dos erros quadrados para o Etanol mostrou-se ainda menor do que o apresentado para o ácido benzoico, com um valor de $8,09 \times 10^{-5}$, validando a acurácia do programa desenvolvido. Assim como nas demais substâncias apresentadas, os valores de Pressão de vapor do Ácido Acético obtidos pelo programa desenvolvido no MS Excel apresentam-se muito próximos dos valores calculados a partir da Equação de Antoine, tomada como padrão para a validação do programa.

O programa mostrou ser capaz de predizer a pressão de vapor das três substâncias analisadas. Isto demonstra que a utilização deste programa pode ser uma forma de calcular esta propriedade para estas substâncias de forma interativa e eficaz com o uso de uma ferramenta acessível como o Software Microsoft Excel.

\section{CONSIDERAÇÕES FINAIS}

O presente estudo apresenta o Excel como um software útil para desenvolver ferramentas de alta capacidade de reprodutibilidade de resolução de equações. O programa desenvolvido demonstra uma alternativa rápida e eficaz para a resolução de equações cúbicas e eficiente para o cálculo da pressão de vapor de espécies químicas e confirma que a EdE de Peng-Robinson pode ser aplicada para calcular a pressão de vapor e modelar o comportamento de espécies químicas com boa precisão.

Por seu fácil manuseio e apresentar respostas próximas de dados experimentais ao comparar com a equação empírica de Antoine. O programa desenvolvido pode auxiliar os pesquisadores a simularem condições de processos nos quais estarão trabalhando.

\section{REFERÊNCIAS}


ASSELINEAU, L., G. BOGDANIC, e J. VIDAL, "Calculation of Thermodynamic Properties and Vapour-Liquid Equilibria of Refrigerants,'” Chem. Eng. Sci., 1978.

ATKINS, P. W.; PAULA, Julio de. Físico-química. 8. ed. Rio de Janeiro: LTC, 2008. 2 v. ISBN 9788521616009 (v.1).

BOUBLIK, T., "Statistical Thermodynamics of Nonspherical Molecule Fluids," Ber. Bunsenges. Phys. Chem., 1981.

CARNAHAN, N. F., e K. E. STARLING, "Equation of State for Nonattracting Rigid Spheres,'” J. Chem. Phys., 1969.

CHEN, S. S., e A. KREGLEWSKI, "Applications of the Augmented van der Waals Theory of Fluids: I. Pure Fluids,’’ Ber. Bunsen-Ges. Phys.Chem., 1977.

CHRISTOFORAKOS, M., e E. U. FRANCK, “An Equation of State for Binary Fluid Mixtures to High Temperatures and High Pressures,' 'Ber. Bunsenges. Phys. Chem., 1986.

DIEFENBACHER, A. e TÜRK, M. Critical properties (pc, Tc, and $\rho c$ ) and phase equilibria of binary mixtures of CO2, CHF3, CH2F2, and SF6.Fluid Phase Equilibria. Volume 182, 2001.

ECONOMOU, I. G., e M. D. DONOHUE, "Equations of State for Hydrogen Bonding Systems,' Fluid Phase Equilib., 1996.

GEANKOPLIS, C. J. Transport Processes and Unit Operations. Prentice-Hall International, 1993.

GRABOSKI, M. S., e T. E. DAUBERT, "A Modified Soave Equation of State for Phase Equilibrium Calculations,'” Ind. Eng. Chem. Process. Des. De“., 1978.

HEILIG, M., e E. U. FRANCK, "Calculation of Thermodynamic Properties of Binary Fluid Mixtures to High Temperatures and High Pressures,' Ber. Bunsenges. Phys. Chem., 1989.

HRNČIČ, M. K., CÖR, D., VERBOTEN, M. T. e KNEZ, Z. Application of supercritical and subcritical fluids in food processing. Food Quality and Safety, Volume 2, 2018.

HURON, M. J., D. N. DUFOUR, e J. VIDAL, "Vapour-Liquid Equilibrium and Critical Locus Curve Calculations with Soave Equation for Hydrocarbon Systems with Carbon Dioxide and Hydrogen Sulfide,', Fluid Phase Equilib., 1978.

MARTIN, P. A. Aplicação de técnicas de controle preditivo em uma coluna de destilação. 2011, 175p. Tese de Doutorado, Universidade de São Paulo, Escola Politécnica. São Paulo-SP.

PENG, D. Y., e D. B. ROBINSON, “A New Two-Constant Equation of State,' Ind. Eng. Chem. Fundam., 1976.

POLING, B. E., PRAUSNITZ J. M. e O'CONNELL, J. P. The properties of gases and liquids, $5^{\text {a }}$ edição. McGrall-Hill, 2001.

REDLICH, O., e J. N. S. KWONG, " On the Thermodynamics of Solutions. V: An Equation of State. Fugacities of Gaseous Solutions,'” Chem. Rev., 1949.

SADUS, R. J., "Calculating Critical Transitions of Fluid Mixtures: Theory vs. Experiment," AIChE J., 1994.

SANDLER, S. I., ed., Models for Thermodynamic and Phase Equilibria Calculations, Marcel Dekker, New York, 1994. 
SMITH, J. M., VAN NESS, H. C. e ABBOTT, M. M., Introdução à Termodinâmica da Engenharia Química, 7a Ed., LTC - Livros Técnicos e Científicos Editora, Rio de Janeiro, 2007.

SOAVE, G., "Equilibrium Constants from a Modified Redlich-Kwong Equation of State," Chem. Eng. Sci., 1972. 